\title{
Stability of a diffusive predator-prey model with modified Leslie-Gower and Holling-type II schemes and time-delay in two dimensions
}

\author{
Aka Fulgence Nindjin ${ }^{1}$, Kessé Thiban Tia ${ }^{1}$, Hypolithe Okou ${ }^{1 *}$ and Albin Tetchi ${ }^{1}$
}

*Correspondence:

okouakpetihi@hotmail.com 'UFR de Mathématiques et Informatique, Université Félix Houphouët Boigny, Abidjan, Côte d'Ivoire

\begin{abstract}
Our investigation deals with the stability and permanence of diffusive predator-prey model with modified Leslie-Gower and Holling-type II schemes and time-delay in two dimensions. Firstly, we prove that the solutions of this model are globally bounded and remain permanently in the positive quadrant. From this system, we obtain three trivial equilibrium points of which, under certain conditions, one is locally stable and the others unstable. We show that the unique point of positive internal equilibrium is locally stable. Then, by constructing an appropriate Lyapunov's functional, we establish the main result which is the global and asymptotic stability of this model. Finally, a numerical simulation is run to illustrate all these different theoretical results.
\end{abstract}

MSC: $92 \mathrm{D} 25 ; 35 \mathrm{~B} 35 ; 35 \mathrm{~B} 38 ; 34 \mathrm{~N} 05$

Keywords: Holling-2; Leslie-Gower; boundedness; permanence; Lyapunov's functional; equilibrium; stability; local; global; delay

\section{Model}

In recent decades, diffusion reaction systems with prey-predator interactions have been extensively studied by the authors such as Camara et al. [1]. They were interested in the following model of Leslie-Gower and Holling II modified in two dimensions:

$$
\begin{cases}\frac{\partial H(T, X)}{\partial T}=\delta_{1} \Delta H(T, X)+\left(a_{1}-b_{1} H(T, X)-\frac{c_{1} P(T, X)}{H(T, X)+k_{1}}\right) H(T, X), & T>0, X \in \Omega, \\ \frac{\partial P(T, X)}{\partial T}=\delta_{2} \Delta P(T, X)+\left(a_{2}-\frac{c_{2} P(T, X)}{H(T, X)+k_{2}}\right) P(T, X), & T>0, X \in \Omega, \\ H(0, X)=H_{0}(X), P(0, X)=P_{0}(X), & \\ \frac{\partial H}{\partial n}=\frac{\partial P}{\partial n}=0, & \text { on } \partial \Omega .\end{cases}
$$

$H$ denotes the density of the prey and $P$ is the density of the predator at time $T, \frac{d H}{d T}$ and $\frac{d P}{d T}$ respectively represent the rate of prey increase and of predators at time $T$ which depend on the following ecological parameters: $a_{1}$ the prey birth rate, $b_{1}$ the mortality rate due to internal prey competition, $c_{1}$ the maximum predation rate, $k_{1}$ the rate of prey protection

(c) The Author(s) 2018. This article is distributed under the terms of the Creative Commons Attribution 4.0 International License (http://creativecommons.org/licenses/by/4.0/), which permits unrestricted use, distribution, and reproduction in any medium, provided you give appropriate credit to the original author(s) and the source, provide a link to the Creative Commons license, and indicate if changes were made. 
by nature, $a_{2}$ the predator birth rate, $c_{2}$ the maximum predator mortality rate, $k_{2}$ the protection rate of predators by nature, $\delta_{2}$ the predator diffusion coefficient, $\Delta$ is the Laplace operator.

In their work, they proved the existence, roundedness, and permanence of solutions in the positive quadrant. Then they showed that the dynamics studied are locally, globally, and asymptotically stable. Few years ago, Nindjin et al. [2] were interested in this model but with several constant arguments delayed and without the term of diffusion. Other authors such as Yanling Tian and Peixuan Weng [3], Yanling Tian and Guangzhou [4] looked at the model [1] with introduction of delays in Leslie-Gower terms. They used the method of upper and lower solution to show the global and asymptotic stability around the equilibrium points. In these works [2-4], the delays are in most cases put in the predator's functional response to prey or in the negative feedback of predator density. The delays used in these papers are often due to the hunting of the predator and the gestation of the prey.

In our paper, we study the impact of internal competition between prey on species dynamics in the presence. For this, we consider the model (studied in [1]) of Leslie-Gower and Holling II modified in two dimensions with diffusion in which we introduce a delay. Taking into account the fact that the internal competition between the individuals of the prey species for the search for food involves the individuals having reached an appropriate maturity, we introduce a delay $r_{1}$ into the prey equation, precisely in the negative feedback of the prey. This delay defines a time of recruitment, that means the necessary time for the immature prey to move into the class of mature prey participating in hunting, procreation, in a word, to the dynamics of the species present and in interaction.

Thus, taking into account the following variable changes:

$$
\begin{aligned}
& t=a_{1} T, \quad x=\left(\frac{a_{1}}{\delta_{1}}\right)^{\frac{1}{2}} X, \\
& u(t, x)=\frac{b_{1}}{a_{1}} H(T, X), \quad v(t, x)=\frac{b_{1} c_{2}}{a_{1} a_{2}} P(T, X), \\
& a=\frac{a_{2} c_{1}}{a_{1} c_{2}}, \quad b=\frac{a_{2}}{a_{1}}, \quad \delta=\frac{\delta_{2}}{\delta_{1}}, \quad e_{1}=\frac{k_{1} b_{1}}{a_{1}}, \quad e_{2}=\frac{k_{2} b_{1}}{a_{1}},
\end{aligned}
$$

we obtain the following model:

$$
\begin{cases}\frac{\partial u(t, x)}{\partial t}=\Delta u(t, x)+\left(1-u\left(t-r_{1}, x\right)-\frac{a v(t, x)}{u(t, x)+e_{1}}\right) u(t, x), & x \in \Omega, t>0, \\ \frac{\partial v(t, x)}{\partial t}=\delta \Delta v(t, x)+b v(t, x)\left(1-\frac{v(t, x)}{u(t, x)+e_{2}}\right), & x \in \Omega, t>0, \\ \left(u_{0}, v_{0}\right)=\phi=\left(\phi_{1}, \phi_{2}\right) \in C\left(\left[-r_{1} ; 0\right] \times \bar{\Omega} ; \mathbb{R}^{2}\right), & \\ u_{0}(\theta, x)=\phi_{1}(\theta, x) \geq 0 \text { and } v_{0}(\theta, x)=\phi_{2}(\theta, x) \geq 0, & x \in \Omega, \theta \in\left[-r_{1} ; 0\right], \\ u(0, x)>0 \text { and } \quad v(0, x)>0, & \text { on } I \times \partial \Omega . \\ \frac{\partial u}{\partial n}=\frac{\partial v}{\partial n}=0, & \end{cases}
$$

In the study of (2), we were inspired by the method used in [4], to show the existence and global boundedness of solutions. Then we study the local stability and permanence of the system. Our goal in this article is to find natural conditions on the control parameters, realistic and easily verifiable, under which the overall stability of our system exists, when 
the length of the delay $r_{1}$ is small enough. To reach this goal, we have developed a method which is a combination of the one used in [2] and [1] to build an appropriate Lyapunov's functional. Definitely, a numerical simulation is run to illustrate all these different theoretical results.

\section{Global boundedness}

\subsection{Existence of solution}

The existence of solutions of the model is done by referring to Theorem 2.1 of [5].

\subsection{Global boundedness}

\section{Theorem 2.1 If}

$$
1-\frac{a}{e_{1}}\left(e^{r_{1}}+e_{2}\right)>0
$$

then the system is globally bounded and, for every positive $\epsilon$ very small, any solution to problem (2) remains in the following domain of $\mathbb{R}_{+}^{*} \times \mathbb{R}_{+}^{*}$ :

$$
\left[\left(1-\frac{a}{e_{1}}\left(e^{r_{1}}+e_{2}\right)\right) e^{r_{1}\left(1-\frac{a}{e_{1}}\left(e^{r_{1}}+e_{2}\right)\right)-r_{1} e^{r_{1}}-r_{1} \epsilon} ; e^{r_{1}}+\epsilon\right] \times\left[e_{2}-\epsilon ; \epsilon+e^{r_{1}}+e_{2}\right] .
$$

Proof Let us consider the first equation of (2). We obtain the following majoration:

$$
\frac{\partial u(t, x)}{\partial t}-\Delta u(t, x) \leq\left(1-u\left(t-r_{1}, x\right)\right) u(t, x) .
$$

Then we add the Neumann condition $\frac{\partial u}{\partial n}=0$ on $\left[-r_{1} ;+\infty[\times \partial \Omega\right.$.

Let us consider the following differential equation:

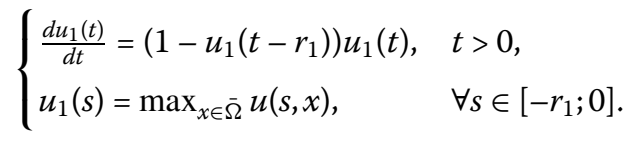

By solving (5), we show that $\ln \frac{u_{1}(t)}{u_{1}\left(t-r_{1}\right)}=\int_{0}^{r_{1}}\left(1-u_{1}\left(t-s-r_{1}\right)\right) d s \leq r_{1}$.

So, $e^{-r_{1}} u_{1}(t) \leq u_{1}\left(t-r_{1}\right)$, which leads to $\frac{d u_{1}(t)}{d t} \leq\left(1-e^{-r_{1}} u_{1}(t)\right) u_{1}(t)$. Thus, by applying one of the consequences of the Gronwall lemma, we have $u_{1}(t) \leq \frac{e^{r_{1}}}{1+\left(\frac{e^{\prime} 1}{u_{1}(0)}-1\right) e^{-t}}$. Hence $\lim \sup _{t \rightarrow+\infty}\left(\max _{x \in \bar{\Omega}} u(t, x)\right) \leq e^{r_{1}}$.

Therefore, $\forall \epsilon_{1}>0$, there is $T_{1}>0$ such that $u(t, x) \leq e^{r_{1}}+\epsilon_{1}$ for $t>T_{1}$ and $x \in \Omega$. From the second equation of (2), we have $\frac{\partial v(t, x)}{\partial t}-\delta \Delta v(t, x) \leq b v(t, x)\left(1-\frac{v(t, x)}{e^{r} 1+\epsilon+e_{2}}\right)$. Let us consider the following problem $\frac{d v_{2}(t)}{d t}=b v_{2}(t)\left(1-\frac{v_{2}(t)}{\epsilon_{1}+e^{1} 1+e_{2}}\right)$ with $v_{2}(s)=\max _{x \in \bar{\Omega}} v(s, x) \forall s \in\left[-r_{1} ; 0\right]$.

So, $v_{2}(t)=\frac{1}{\frac{1}{\epsilon_{1}+e^{r_{1}}+e_{2}}+k e^{-b t}}, k \in \mathbb{R}^{+}$. Hence lim $\left.\sup _{t \rightarrow+\infty} \max _{\bar{\Omega}} v(t, x)\right) \leq \epsilon_{1}+e^{r_{1}}+e_{2}$.

When $\epsilon_{1}$ tends to zero, we get lim $\left.\sup _{t \rightarrow+\infty} \max _{\bar{\Omega}} v(t, x)\right) \leq e^{r_{1}}+e_{2}$.

Therefore, $\forall \epsilon_{2}>0$ there is $T_{2}>T_{1}$ such that

$$
v(t, x)) \leq \epsilon_{2}+e^{r_{1}}+e_{2}, \quad \forall t>T_{2}
$$

Determination of the lower limit of $u$ and $v$.

We have $b v(t, x)\left(1-\frac{v(t, x)}{e_{2}}\right) \leq \frac{\partial v(t, x)}{\partial t}-\delta \Delta v(t, x)$. 
Let us consider the following problem:

$$
\frac{d v_{3}(t)}{d t}=b v_{3}(t)\left(1-\frac{v_{3}(t)}{e_{2}}\right) \quad \text { with } v_{3}(s)=\min _{\bar{\Omega}} v(s, x), s \in\left[-r_{1} ; 0\right] .
$$

Solving this problem, we have $v_{3}(t)=\frac{1}{\frac{1}{e_{2}}+k e^{-b t}}$, so, $\lim _{t \rightarrow+\infty} v_{3}(t)=e_{2}$.

Hence $\forall \epsilon_{3}>0, \exists T_{3}>0$ so that $\forall t>T_{3}, v_{3}(t) \geq e_{2}-\epsilon_{3}$.

Thus, $\left.\liminf _{t \rightarrow+\infty} \min _{\bar{\Omega}} v(t, x)\right) \geq e_{2}$.

Let us show that $u$ is greater than a strictly positive real.

We know that $\left.\limsup _{t \rightarrow+\infty} \max _{\bar{\Omega}} v(t, x)\right) \leq e^{r_{1}}+e_{2}=\eta$ and $\left.\limsup _{t \rightarrow+\infty} \max _{\bar{\Omega}} u(t, x)\right) \leq$ $e^{r_{1}}$.

Therefore, $\forall \epsilon_{3}>0$ there is $T_{3}>T_{2}$ such that $\left.v(t, x)\right) \leq \eta+\epsilon_{3}, \forall t>T_{3}$.

Using this relation, we show that

$$
\left\{\begin{array}{l}
\frac{\partial u(t, x)}{\partial t}-\Delta u(t, x) \geq\left(K-u\left(t-r_{1}, x\right)\right) u(t, x), \\
\quad \text { with } K=1-\frac{a\left(e^{r_{1}}+e_{2}+\epsilon_{3}\right)}{e_{1}},
\end{array}\right.
$$

and consider the following system:

$$
\left\{\begin{array}{l}
\frac{d u_{2}(t)}{d t}=u_{2}(t)\left(K-u_{2}\left(t-r_{1}\right)\right), \\
u_{2}(s)=\min u(s, x), \quad(s, x) \in[-r ; 0] \times \bar{\Omega} .
\end{array}\right.
$$

We know that $\forall t>T_{3}, u(t, x) \leq e^{r_{1}}+\epsilon$, so $\forall t>T_{3}+r_{1}, u\left(t-r_{1}, x\right) \leq e^{r_{1}}+\epsilon$, hence $u_{2}(t-$ $\left.r_{1}\right) \leq e^{r_{1}}+\epsilon$.

By injecting into system (8), we have $\frac{d u_{2}(t)}{u_{2}(t} \geq \beta d t$, with $\beta=K-e^{r_{1}}-\epsilon$.

Taking the integral in this inequality between $t$ and $t-r_{1}$, we have $u_{2}\left(t-r_{1}\right) \leq e^{-\beta r_{1}} u_{2}(t)$. Taking into account system (8), we obtain

$$
\frac{d u_{2}(t)}{d t} \geq\left(K-e^{-\beta r_{1}} u_{2}(t)\right) u_{2}(t)
$$

When we pass to the integral in (9) between $s \in[-r ; 0]$ and $t>0$, we have $u_{2}(t) \geq \frac{K}{\gamma e^{-K t}+e^{-\beta r_{1}}}$. Thus,

$$
\liminf _{t \rightarrow+\infty} u_{2}(t) \geq K e^{\beta r_{1}}
$$

Finally, we have

$$
\left.\liminf _{t \rightarrow+\infty} \min _{\bar{\Omega}} u(t, x)\right) \geq\left[1-\frac{a}{e_{1}}\left(e^{r_{1}}+e_{2}\right)\right] e^{r_{1}\left[1-\frac{a}{e_{1}}\left(e^{r_{1}}+e_{2}\right)\right]-r_{1} e^{r_{1}}} .
$$

Therefore, this ensures the global boundedness.

Remark 2.1 We note that hypothesis (3) of the theorem is equivalent to

$$
e_{1}-a e_{2}>a \quad \text { and } \quad r_{1} \in\left[0 ; \ln \left(\frac{e_{1}-a e_{2}}{a}\right)\right]
$$


Thus, condition (10) has the advantage of giving the delay interval. So, the values taken by the parameters $e_{1}, e_{2}$, and $a$ determine the existence and the length of the delay $r_{1}$ able to ensure the boundedness of the solutions.

\section{The study of the local stability of the system}

Let us recall that the delay system has the same equilibrium points as the system without delay in [1]. It is clear that the trivial equilibrium points are: $S_{0}=(0,0), S_{1}=\left(0, e_{2}\right), S_{2}=$ $(1,0)$. As for the existence of the positive internal fixed point $S_{3}=\left(u^{*}, v^{*}\right)$, we have the following theorem.

Theorem 3.1 If $e_{1}>a e_{2}$, then the system admits a unique interior fixed point $S_{3}=\left(u^{*}, v^{*}\right)$, $u^{*}=\frac{1-a-e_{1}+\sqrt{\left(a-1+e_{1}\right)^{2}+4\left(e_{1}-a e_{2}\right)}}{2}$ and $v^{*}=u^{*}+e_{2}$.

Proof System (2) admits a constant internal equilibrium point $S_{3}=\left(u^{*}, v^{*}\right)$ only if $\left(u^{*}, v^{*}\right)$ is the solution of the following system:

$$
\left\{\begin{array}{l}
1-u^{*}-\frac{a v^{*}}{u^{*}+e_{1}}=0 \\
1-\frac{v^{*}}{u^{*}+e_{2}}=0
\end{array}\right.
$$

Considering the first equation of system (11), we obtain

$$
1-u^{*}-\frac{a v^{*}}{u^{*}+e_{1}}=0 \quad \Longleftrightarrow \quad v^{*}=\frac{1}{a}\left(-u^{* 2}+\left(1-e_{1}\right) u^{*}+e_{1}\right) .
$$

Replacing $v^{*}$ by $\frac{1}{a}\left(-u^{* 2}+\left(1-e_{1}\right) u^{*}+e_{1}\right)$ in the second equation, we obtain $u^{* 2}+(a-1+$ $\left.e_{1}\right) u^{*}+e_{2} a-e_{1}=0$ whose resolution gives

$$
u_{1}^{*}=\frac{1-a-e_{1}+\sqrt{\left(a-1+e_{1}\right)^{2}+4\left(e_{1}-a e_{2}\right)}}{2}
$$

and

$$
u_{2}^{*}=\frac{1-a-e_{1}-\sqrt{\left(a-1+e_{1}\right)^{2}+4\left(e_{1}-a e_{2}\right)}}{2} .
$$

We show that $u_{1}^{*}>0$ as well as $v_{1}^{*}=u_{1}^{*}+e_{2}$ is.

We study the local stability in the neighborhood of $S_{i}$ where $i=0,1,2,3$. Let us consider the functional

$$
\begin{aligned}
& F: \mathbb{R}^{4} \rightarrow \mathbb{R}^{2}, \\
& \left(u(t, x), v(t, x), u\left(t-r_{1}, x\right), v\left(t-r_{1}, x\right)\right) \mapsto\left(\begin{array}{c}
u(t, x)\left(1-u\left(t-r_{1}, x\right)-\frac{a v(t, x)}{u(t, x)+e_{1}}\right) \\
b v(t, x)\left(1-\frac{v(t, x)+e_{2}}{u(t, x)+e_{2}}\right) .
\end{array}\right.
\end{aligned}
$$

Thus, the Jacobian matrix of $F$ is

$$
J_{F}\left(u(t, x), v(t, x), u\left(t-r_{1}, x\right), v\left(t-r_{1}, x\right)\right)=\left(\begin{array}{cccc}
A_{11} & A_{12} & A_{13} & 0 \\
A_{21} & A_{22} & 0 & 0
\end{array}\right)
$$


with $A_{11}=1-u\left(t-r_{1}, x\right)-\frac{a v(t, x)}{u(t, x)+e_{1}}+a u(t, x) \frac{v(t, x)}{\left(u(t, x)+e_{1}\right)^{2}}, A_{12}=-\frac{a u(t, x)}{u(t, x)+e_{1}}, A_{13}=-u(t, x), A_{21}=$ $\frac{b v^{2}(t, x)}{\left(u(t, x)+e_{2}\right)^{2}}$ and $A_{22}=b\left(1-2 \frac{v(t, x)}{u(t, x)+e_{2}}\right)$.

System (2) can be written in the form

$$
\frac{\partial}{\partial t}\left(\begin{array}{l}
u(t, x) \\
v(t, x)
\end{array}\right)=\left(\begin{array}{cc}
\Delta & 0 \\
0 & \delta \Delta
\end{array}\right)\left(\begin{array}{l}
u(t, x) \\
v(t, x)
\end{array}\right)+F\left(u(t, x), v(t, x), u\left(t-r_{1}, x\right), v\left(t-r_{1}, x\right)\right)
$$

Let us pose: $w=\left(\begin{array}{c}w_{1} \\ w_{2}\end{array}\right)=\left(\begin{array}{l}u \\ v\end{array}\right)-S_{i}$. Let us note $S_{i}^{*}$ the fixed point taking into account the delay corresponding to $\left(u(t, x), v(t, x), u\left(t-r_{1}, x\right), v\left(t-r_{1}, x\right)\right)$.

So, the system linearized around $S_{i}$ becomes

$$
\frac{\partial}{\partial t}\left(\begin{array}{l}
w_{1}(t, x) \\
w_{2}(t, x)
\end{array}\right)=\left(\begin{array}{cc}
\Delta & 0 \\
0 & \delta \Delta
\end{array}\right)\left(\begin{array}{l}
w_{1}(t, x) \\
w_{2}(t, x)
\end{array}\right)+J_{F}\left(S_{i}^{*}\right)\left(\begin{array}{c}
w_{1}(t, x) \\
w_{2}(t, x) \\
w_{1}\left(t-r_{1}, x\right) \\
w_{2}\left(t-r_{1}, x\right)
\end{array}\right)
$$

which is equivalent to the following system:

$$
\left\{\begin{array}{l}
\frac{\partial w_{1}(t, x)}{\partial t}=\Delta w_{1}(t, x)+A_{11} w_{1}(t, x)+A_{12} w_{2}(t, x)+A_{13} w_{1}\left(t-r_{1}, x\right) \\
\frac{\partial w_{2}(t, x)}{\partial t}=\delta \Delta w_{2}(t, x)+A_{21} w_{1}(t, x)+A_{22} w_{2}(t, x)
\end{array}\right.
$$

\subsection{Local stability of $S_{0}$ and $S_{1}$}

For the points $S_{0}=(0 ; 0)$ and $S_{1}=\left(0 ; e_{2}\right)$, we have $A_{13}=0$. Then everything happens as if we are in the case of a model without delay. Now, for the model without delay studied in [2], we have the following conclusions:

(i) $S_{0}=(0 ; 0)$ is unstable.

(ii) $S_{1}=\left(0 ; e_{2}\right)$ is stable if $e_{1}<a e_{2}$, and unstable if $e_{1}>a e_{2}$.

It can be concluded that the delay $r_{1}$ had no influence on the stability of the equilibrium points $S_{0}$ and $S_{1}$.

\subsection{Local stability of $S_{2}$ and $S_{3}$}

For the equilibria points $S_{2}$ and $S_{3}$, we have $A_{13} \neq 0$.

In order to determine the characteristic equation of model (2), we consider $\left(\mu_{i}, \varphi_{i}\right)_{i=0}^{\infty}$ the set of value and eigenvector pairs of the $-\Delta$ operator on $\bar{\Omega}=\left[0 ; M_{x}\right] \times\left[0 ; M_{y}\right]$ with homogeneous Neumann type boundary conditions such as

$$
0=\mu_{0}<\mu_{1}<\mu_{2}<\cdots \quad \text { with } \mu_{i}=\pi^{2}\left(\frac{p_{i}^{2}}{M_{x}^{2}}+\frac{q_{i}^{2}}{M_{y}^{2}}\right), p_{i}, q_{i} \in \mathbb{N} .
$$

Let $X=\left\{(u, v) \in C^{2}(\bar{\Omega}) \times C^{2}(\bar{\Omega}) / \frac{\partial u}{\partial n}=\frac{\partial v}{\partial n}=0\right\}$ be a set that can be decomposed as a direct sum $\bigoplus_{i=0}^{\infty} X_{i}$, where $X_{i}$ is the space of the eigenvectors corresponding to the eigenvalue $\mu_{i}$ for all $i=0 ; 1 ; 2 ; \ldots$.

For each $i=0 ; 1 ; 2 ; \ldots$, the set $X_{i}$ is invariant under operator of (13). So, the characteristic equation of the linearized system of (2) is

$$
P(\lambda)+Q(\lambda) e^{-r_{1} \lambda}=0
$$

where $P(\lambda)=\left(\lambda+\delta \mu_{i}-A_{22}\right)\left(\lambda+\mu_{i}-A_{11}\right)-A_{12} A_{21}$ and $Q(\lambda)=-A_{13}\left(\lambda+\delta \mu_{i}-A_{22}\right)$. 
For all $i=0 ; 1 ; 2 ; \ldots$, we have

1. $\quad P(\lambda)$ and $Q(\lambda)$ have no common imaginary roots because $Q(\lambda)$ has only one root that is real.

2. If $\mu_{i} \neq \frac{b}{\delta}$, then around $S_{2}$ we have $P(0)+Q(0) \neq 0$. If $e_{1}>1$, then around $S_{3}$ one has $P(0)+Q(0) \neq 0$.

3. It is clear that $\overline{P(-i \omega)}=P(i \omega)$ and $\overline{Q(-i \omega)}=Q(i \omega)$.

4. One has $\lim \sup \left\{\left|\frac{Q(\lambda)}{P(\lambda)}\right| /|\lambda| \rightarrow+\infty\right.$ and $\left.\operatorname{Re}(\lambda) \geq 0\right\}=0$. Then $\lim \sup \left\{\left|\frac{Q(\lambda)}{P(\lambda)}\right| /|\lambda| \rightarrow+\infty\right.$ and $\left.\operatorname{Re}(\lambda) \geq 0\right\}<1$.

5. Let us pose $F(\omega)=|P(i \omega)|^{2}-|Q(i \omega)|^{2}$. The function $F$ can be put in the form $F(\omega)=\omega^{4}+m_{1} \omega^{2}+m_{0}$, where $m_{0}$ and $m_{1}$ are the real numbers $\omega^{*}$.

6. Let us consider $\omega^{*}=\omega\left(r^{*}\right)$ a possible positive root of $F(\omega)$ and its associated delay $r_{0}=\left[\frac{1}{\omega^{*}} \arctan \left\{-\frac{\operatorname{Im}\left(\frac{P\left(i \omega^{*}\right)}{Q\left(i \omega \omega^{*}\right)}\right)}{\operatorname{Re}\left(\frac{\left.P(i)^{*}\right)}{Q\left(i \omega^{*}\right)}\right)}\right\}+\frac{2 n \pi}{\omega^{*}}\right], n \in \mathbb{N}$. So, there are $\operatorname{Sgn}\left(\frac{d \operatorname{Re}(\lambda)}{d r}\right)_{\lambda=i \omega^{*}}=\operatorname{Sgn}\left(F^{\prime}\left(\omega^{*}\right)\right)$.

3.2.1 Around $S_{2}=(1 ; 0)$

One has $A_{11}=0, A_{12}=-\frac{a}{1+e_{1}}, A_{13}=-1, A_{21}=0, A_{22}=b, m_{0}=\left(\mu_{i}^{2}-1\right)\left(\delta \mu_{i}-b\right)^{2}$ and $m_{1}=$ $\mu_{i}^{2}-1+\left(\delta \mu_{i}-b\right)^{2}$. Therefore:

If $m_{0}>0$, then $m_{1}>0$. So, $F$ does not admit a positive solution. So, we have no stability change. In this case, as at $r_{1}=0$, the system is unstable, it remains so.

If $m_{0}<0$, then $F$ has exactly one positive solution $\omega^{*}$. Now

$$
\operatorname{Sgn}\left(\frac{d \operatorname{Re}(\lambda)}{d r}\right)_{\lambda=i \omega^{*}}=\operatorname{Sgn}\left(F^{\prime}\left(\omega^{*}\right)\right)=\operatorname{Sgn}\left(\left(\omega^{*}\right)^{4}-m_{0}\right)>0 .
$$

So, it does not have any change of stability. In a way similar to the previous case, the system is unstable.

\subsubsection{Around $S_{3}=\left(u^{*}, v^{*}\right)$}

One has $A_{11}=\frac{a u^{*} v^{*}}{\left(u^{*}+e_{1}\right)^{2}}, A_{12}=-\frac{a u^{*}}{u^{*}+e_{1}}, A_{13}=-u^{*}, A_{21}=b, A_{22}=-b, m_{0}=\left[A_{21} A_{12}-\left(\mu_{i}-\right.\right.$ $\left.\left.A_{11}\right)\left(\delta \mu_{i}-A_{22}\right)\right]^{2}-A_{13}^{2}\left(\delta \mu_{i}-A_{22}\right)^{2}$, and $m_{1}=-A_{13}^{2}+2 A_{21} A_{12}-2\left(\mu_{i}-A_{11}\right)\left(\delta \mu_{i}-A_{22}\right)+$ $\left(\mu_{i}-A_{11}-A_{22}+\delta \mu_{i}\right)^{2}$.

- If $m_{1}>0$ and $m_{0}>0$, then $F$ does not admit any positive root. In this case, there is no change in stability. Now at $r_{1}=0$, in [1], if $a \geq \frac{1}{2}$ and $e_{1}>-(a+1)+\sqrt{(a+1)^{2}+2 a\left(1+2 e_{2}\right)-1}$, then the equilibrium point $S_{3}=\left(u^{*}, v^{*}\right)$ is stable. Therefore, it remains so.

- If $m_{0}<0$, then $F$ has a single positive root. There is a positive real $r_{0}$ beyond which there is a change of stability.

Remark 3.1 Considering certain conditions, we can come up with very practical cases. So, to have $m_{1}>0$ and $m_{0}>0$, just take for example $\mu_{i}>\max \left\{1+e_{1} ; \sqrt{\frac{2 a b u^{*}}{u^{*}+e_{1}}}+\frac{\left(1-u^{*}\right) u^{*}}{u^{*}+e_{1}} ; \frac{1}{\delta}\left(u^{*}-b\right)\right\}$ for each $i=1,2,3, \ldots$.

Similarly, taking $e_{1}>1$ and $\left[-A_{22}+\delta\left(A_{13}-A_{11}\right)\right]^{2}+4\left(A_{13}-A_{11}\right) A_{22}+4 A_{21} A_{12}>0$, we can find $\mu_{i}$ which allow us to have $m_{0}<0$.

\section{Permanence}

Theorem 4.1 If $e_{1}>a e_{2}$, then the system is permanent. 
Proof The trivial points $(0,0)$ and $(1,0)$ are unstable. And if $e_{1}>a e_{2}$, then $\left(0, e_{2}\right)$ is unstable. Thus, the points of equilibrium situated on the axes are all unstable; they cannot attract the other solutions of the system. In addition to this, the solutions of the system are bounded globally, that is to say,

$$
\begin{aligned}
& \forall \epsilon>0, \exists T>0 \text { so that } \forall t>T \text { and } \forall x \in \Omega, \text { we have } \\
& 0<\left[1-\frac{a}{e_{1}}\left(e^{r_{1}}+e_{2}\right)\right] e^{r_{1}\left[1-\frac{a}{e_{1}}\left(e^{r_{1}}+e_{2}\right)\right]-r_{1} e^{r_{1}}-r_{1} \epsilon} \leq u(t, x) \leq e^{r_{1}}+\epsilon \\
& 0<e_{2}-\epsilon \leq v(t, x) \leq \epsilon+e^{r_{1}}+e_{2}
\end{aligned}
$$

for $\epsilon$ smallest. So, the system is permanent.

\section{Global stability}

In this section, we are interested in the study of global stability. Using the results of Lyapunov's functional construction in [2], we construct an appropriate Lyapunov's functional to study the global stability of the unique interior equilibrium point $S_{3}$. For this, we state the following lemma.

Lemma 5.1 Let $f$ be a continuous function on $\mathbb{R}$. The function $g: t \mapsto \int_{t-r_{1}}^{t} \int_{y}^{t} f(s) d s d y$ is differentiable on $\mathbb{R}$, and

$$
\forall t \in \mathbb{R}, \quad g^{\prime}(t)=r_{1} f(t)-\int_{t-r_{1}}^{t} f(s) d s
$$

Proof Let $F$ be a primitive of $f$.

We know that $\forall t \in \mathbb{R}, g(t)=\int_{t-r_{1}}^{t} \int_{y}^{t} f(s) d s d y$.

Thus, $g(t)=\int_{t-r_{1}}^{t}(F(t)-F(y)) d y$. So, $g(t)=r_{1} F(t)-\int_{t-r_{1}}^{t} F(y) d y$. When we pass to the derivative of $g$, we get

$$
g^{\prime}(t)=r_{1} F^{\prime}(t)-\left(F(t)-F\left(t-r_{1}\right)\right)=r_{1} f(t)-\int_{t-r_{1}}^{t} f(s) d s .
$$

Theorem 5.1 (Main theorem) Suppose that the bounding condition (3) and the hypothesis

$$
\frac{2 a e_{2}}{e_{2}+1}>e_{1}>\frac{2 e_{2}}{e_{2}^{2}-1} \quad \text { and } e_{2}>1
$$

are verified. Then, for all $r_{1}$ sufficiently small, the interior equilibrium point $S_{3}$ is globally asymptotically stable in $\mathbb{R}^{2}$.

Proof When the condition $1-\frac{a}{e_{1}}\left(e^{r_{1}}+e_{2}\right)>0$ is satisfied, then $e_{1}>a e_{2}$. Thus, the conditions of global bounding imply those of the existence of the point of interior equilibrium.

1. The solutions of model (2) are bounded, and we have

$$
\begin{aligned}
& m_{u}^{\epsilon} \leq u(t, x) \leq M_{u}^{\epsilon} \text { and } \quad m_{v}^{\epsilon} \leq v(t, x) \leq M_{v}^{\epsilon} \quad \text { with } \\
& m_{u}^{\epsilon}=\left[1-\frac{a}{e_{1}}\left(e^{r_{1}}+e_{2}\right)\right] e^{r_{1}\left[1-\frac{a}{e_{1}}\left(e^{r_{1}}+e_{2}\right)\right]-r_{1} e^{r_{1}}-r_{1} \epsilon} ; \\
& M_{u}^{\epsilon}=e^{r_{1}}+\epsilon ; \quad m_{v}^{\epsilon}=e_{2}-\epsilon ; M_{v}^{\epsilon}=\epsilon+e^{r_{1}}+e_{2} .
\end{aligned}
$$


2. Model (2) has a unique interior fixed point $\left(u^{*}, v^{*}\right)$ verifying (11).

Let us consider the function $l$ so that $\forall \epsilon \geq 0, \exists T>0$ such as $\forall t \geq T$ and $x \in \Omega$,

$$
l(u(t, x), v(t, x))=l_{1}(u(t, x), v(t, x))+\Sigma_{1}(t, x)
$$

with

$$
\begin{aligned}
\Sigma_{1}(t, x)= & \left.\int_{t-r_{1}}^{t} \int_{y}^{t} \frac{M_{u}}{2}\left(u\left(s-r_{1}, x\right)-u^{*}\right)^{2}\right) d s d y \\
& +\int_{t-r_{1}}^{t} \int_{y}^{t} \frac{M_{u}}{2 e_{1}}\left(u(s, x)-u^{*}\right)^{2} d s d y+\int_{t-r_{1}}^{t} \int_{y}^{t} \frac{M_{u}}{2 M_{v}^{\epsilon}}\left(v(s, x)-v^{*}\right)^{2} d s d y \\
& +\int_{t-r_{1}}^{t} \frac{r_{1} M_{u}}{2}\left(u(s, x)-u^{*}\right)^{2} d s+\frac{v^{*}}{M_{v}^{\epsilon}} \int_{t-r_{1}}^{t} \int_{y}^{t} \frac{|\nabla u(s, x)|^{2}}{2} d s d y
\end{aligned}
$$

and

$$
l_{1}(u(t, x), v(t, x))=\frac{v^{*}}{M_{v}^{\epsilon}}\left[u-u^{*}-u^{*} \ln \frac{u}{u^{*}}\right]+\frac{v^{*}}{b M_{v}^{\epsilon}}\left[v-v^{*}-v^{*} \ln \frac{v}{v^{*}}\right]
$$

The function $l_{1}$ admits zero for the global minimum reached in $\left(u^{*}, v^{*}\right)$. So, $l(u(t, x)$, $v(t, x)) \geq 0$ with $l\left(u^{*}, v^{*}\right)=0$. Posing

$$
\begin{aligned}
L(u(t, x), v(t, x)) & =\int_{\Omega} l(u(t, x), v(t, x)) d x \\
& =\int_{\Omega} l_{1}(u(t, x), v(t, x)) d x+\int_{\Omega} \Sigma_{1}(t, x) d x
\end{aligned}
$$

Let us show that the function $L$ as constructed is a Lyapunov's functional for system (2).

(i) We have $L\left(u^{*}, v^{*}\right)=0$.

(ii) For any solution $(u, v)$ of (2) of which the initial condition $\left(u_{0}(x), v_{0}(x)\right)$ in the positive quadrant that solution is positive. Therefore, $L(u, v)$ is positive.

(iii) We have to prove the following inequality: $\frac{d L}{d t}<0$.

$$
\frac{d L}{d t}=\int_{\Omega} \frac{\partial l_{1}(u(t, x), v(t, x))}{\partial t} d x+\int_{\Omega} \frac{\partial \Sigma_{1}(t, x)}{\partial t} d x .
$$

Using relation (11), for all $u$ and $v$ sufficiently regular and bounded, system (2) becomes

$$
\left\{\begin{array}{l}
\frac{\partial_{t} u(t, x)}{u(t, x)}=\frac{\Delta u(t, x)}{u(t, x)}-\left[u\left(t-r_{1}, x\right)-u^{*}\right]+\frac{a v(t, x)}{\left(u^{*}+e_{1}\right)\left(u(t, x)+e_{1}\right)}\left(u(t, x)-u^{*}\right)-\frac{a}{u^{*}+e_{1}}\left(v(t, x)-v^{*}\right) \\
\frac{\partial t v(t, x)}{v(t, x)}=\frac{\delta \Delta v(t, x)}{v(t, x)}+\frac{b v(t, x)}{\left(u^{*}+e_{2}\right)\left(u(t, x)+e_{2}\right)}\left(u(t, x)-u^{*}\right)-\frac{b}{u^{*}+e_{2}}\left(v(t, x)-v^{*}\right)
\end{array}\right.
$$

We have

$$
\begin{aligned}
& \int_{\Omega} \frac{\partial l_{1}(u(t, x), v(t, x))}{\partial t} d x \\
& \quad=\int_{\Omega} \frac{\partial l_{1}(u(t, x), v(t, x))}{\partial u(t, x)} \frac{\partial u(t, x)}{\partial t}+\frac{\partial l_{1}(u(t, x), v(t, x))}{\partial v(t, x)} \frac{\partial v(t, x)}{\partial t} d x
\end{aligned}
$$




$$
\begin{aligned}
= & \int_{\Omega}\left[\frac{v^{*}}{M_{v}^{\epsilon}} \frac{u(t, x)-u^{*}}{u(t, x)} \frac{\partial u(t, x)}{\partial t}+\frac{v^{*}}{b M_{v}^{\epsilon}}\left(1-\frac{v^{*}}{v(t, x)}\right) \frac{\partial v(t, x)}{\partial t}\right] d x \\
= & \int_{\Omega}\left[\frac { v ^ { * } } { M _ { v } ^ { \epsilon } } ( u ( t , x ) - u ^ { * } ) \left[\frac{\Delta u(t, x)}{u(t, x)}-\left(u\left(t-r_{1}, x\right)-u^{*}\right)\right.\right. \\
& \left.+\frac{a v}{\left(u^{*}+e_{1}\right)\left(u(t, x)+e_{1}\right)}\left(u(t, x)-u^{*}\right)-\frac{a}{u^{*}+e_{1}}\left(v(t, x)-v^{*}\right)\right] \\
& +\frac{v^{*}}{b M_{v}^{\epsilon}}\left(v(t, x)-v^{*}\right)\left[\frac{\delta \Delta v(t, x)}{v(t, x)}+\frac{b v(t, x)}{\left(u^{*}+e_{2}\right)\left(u(t, x)+e_{2}\right)}\left(u(t, x)-u^{*}\right)\right. \\
& \left.\left.-\frac{b}{u^{*}+e_{2}}\left(v(t, x)-v^{*}\right)\right]\right] d x .
\end{aligned}
$$

Let us pose $\int_{\Omega} \frac{\partial l_{1}(u(t, x), v(t, x))}{\partial t} d x=T_{1}+T_{2}$ with

$$
\begin{aligned}
T_{1}= & \int_{\Omega}\left[\frac { v ^ { * } } { M _ { v } ^ { \epsilon } } ( u ( t , x ) - u ^ { * } ) \left[-\left(u\left(t-r_{1}, x\right)-u^{*}\right)+\frac{a v}{\left(u^{*}+e_{1}\right)\left(u(t, x)+e_{1}\right)}\left(u(t, x)-u^{*}\right)\right.\right. \\
& \left.-\frac{a}{u^{*}+e_{1}}\left(v(t, x)-v^{*}\right)\right]+\frac{v^{*}}{b M_{v}^{\epsilon}}\left(v(t, x)-v^{*}\right)\left[\frac{b v(t, x)}{\left(u^{*}+e_{2}\right)\left(u(t, x)+e_{2}\right)}\left(u(t, x)-u^{*}\right)\right. \\
& \left.\left.-\frac{b}{u^{*}+e_{2}}\left(v(t, x)-v^{*}\right)\right]\right] d x
\end{aligned}
$$

and

$$
T_{2}=\int_{\Omega}\left[\frac{v^{*}}{M_{v}^{\epsilon}}\left(u(t, x)-u^{*}\right) \frac{\Delta u(t, x)}{u(t, x)}+\frac{v^{*}}{b M_{v}^{\epsilon}}\left(v(t, x)-v^{*}\right) \frac{\delta \Delta v(t, x)}{v(t, x)}\right] d x
$$

Transform $T_{2}$. From Green's formula and taking into account the condition from Neumann $\left(\frac{\partial u}{\partial \eta}=\frac{\partial v}{\partial \eta}=0\right)$, we have

$$
\left.T_{2}=-\frac{u^{*} v^{*}}{M_{v}^{\epsilon}} \int_{\Omega} \frac{|\nabla u(t, x)|^{2}}{u(t, x)^{2}} d x-\delta \frac{\left(v^{*}\right)^{2}}{b M_{v}^{\epsilon}} \int_{\Omega} \frac{|\nabla v(t, x)|^{2}}{v(t, x)^{2}}\right] d x
$$

By the relation: $u\left(t-r_{1}, x\right)=u(t, x)-\int_{t-r_{1}}^{t} \frac{\partial u(s, x)}{\partial s} d s, T_{1}$ becomes

$$
\begin{aligned}
T_{1}= & \int_{\Omega}\left[-\frac{v^{*}}{M_{v}^{\epsilon}}\left(u(t, x)-u^{*}\right)^{2}+\frac{v^{*}}{M_{v}^{\epsilon}}\left(u(t, x)-u^{*}\right) \int_{t-r_{1}}^{t} \frac{\partial u(s, x)}{\partial s} d s\right. \\
& +\frac{v^{*} a v}{M_{v}^{\epsilon}\left(u^{*}+e_{1}\right)\left(u(t, x)+e_{1}\right)}\left(u(t, x)-u^{*}\right)^{2} \\
& -\frac{v^{*} a}{M_{v}^{\epsilon}\left(u^{*}+e_{1}\right)}\left(u(t, x)-u^{*}\right)\left(v(t, x)-v^{*}\right) \\
& +\frac{v^{*} v(t, x)}{M_{v}^{\epsilon}\left(u^{*}+e_{2}\right)\left(u(t, x)+e_{2}\right)}\left(u(t, x)-u^{*}\right)\left(v(t, x)-v^{*}\right) \\
& \left.-\frac{v^{*}}{M_{v}^{\epsilon}\left(u^{*}+e_{2}\right)}\left(v(t, x)-v^{*}\right)^{2}\right] d x .
\end{aligned}
$$


So, $T_{1}$ must be decomposed into a sum of two large terms which we denote by $T_{11}$ and $T_{12}$, where

$$
\begin{aligned}
T_{11}= & \int_{\Omega}\left[\left[-\frac{v^{*}}{M_{v}^{\epsilon}}+\frac{v^{*} a v(t, x)}{M_{v}^{\epsilon}\left(u^{*}+e_{1}\right)\left(u(t, x)+e_{1}\right)}\right]\left(u(t, x)-u^{*}\right)^{2}\right. \\
& +\left[-\frac{v^{*} a}{M_{v}^{\epsilon}\left(u^{*}+e_{1}\right)}+\frac{v^{*} v(t, x)}{M_{v}^{\epsilon}\left(u^{*}+e_{2}\right)\left(u(t, x)+e_{2}\right)}\right]\left(u(t, x)-u^{*}\right)\left(v(t, x)-v^{*}\right) \\
& \left.-\frac{v^{*}}{M_{v}^{\epsilon}\left(u^{*}+e_{2}\right)}\left(v(t, x)-v^{*}\right)^{2}\right] d x
\end{aligned}
$$

and $T_{12}=\int_{\Omega} \frac{v^{*}}{M_{v}^{\epsilon}}\left(u(t, x)-u^{*}\right) \int_{t-r_{1}}^{t} \frac{\partial u(s, x)}{\partial s} d s d x$.

Using (11), let us compute the supremum value of $T_{11}$ and $T_{12}$. So,

$$
\begin{aligned}
T_{11}= & \int_{\Omega}\left[-\frac{v^{*}}{M_{v}^{\epsilon}}+\frac{\left(1-u^{*}\right) v(t, x)}{M_{v}^{\epsilon}\left(u(t, x)+e_{1}\right)}\right]\left(u(t, x)-u^{*}\right)^{2} d x \\
& +\int_{\Omega}\left[\left(-\frac{\left(1-u^{*}\right)}{M_{v}^{\epsilon}}+\frac{v}{M_{v}^{\epsilon}\left(u(t, x)+e_{2}\right)}\right)\left(u(t, x)-u^{*}\right)\left(v(t, x)-v^{*}\right)\right. \\
& \left.-\frac{1}{M_{v}^{\epsilon}}\left(v(t, x)-v^{*}\right)^{2}\right] d x \\
\Longrightarrow & \\
T_{11} \leq & \int_{\Omega}\left[-\frac{m_{v}^{\epsilon}}{M_{v}^{\epsilon}}+\frac{1}{e_{1}}\right]\left(u(t, x)-u^{*}\right)^{2} d x \\
& +\int_{\Omega}\left[\left|\left(-\frac{\left(1-u^{*}\right)}{M_{v}^{\epsilon}}+\frac{v}{M_{v}^{\epsilon}\left(u(t, x)+e_{2}\right)}\right)\right|\left(u(t, x)-u^{*}\right)\left(v(t, x)-v^{*}\right) \mid\right. \\
& \left.-\frac{1}{M_{v}^{\epsilon}}\left(v(t, x)-v^{*}\right)^{2}\right] d x .
\end{aligned}
$$

Hence

$$
\begin{aligned}
T_{11} \leq & \int_{\Omega}\left[-\frac{e_{2}}{M_{v}^{\epsilon}}+\frac{1}{e_{1}}+\frac{1}{2}+\frac{1}{2 e_{2}}\right]\left(u(t, x)-u^{*}\right)^{2} d x \\
& \left.+\int_{\Omega}\left[-\frac{1}{M_{v}^{\epsilon}}+\frac{1}{2}+\frac{1}{2 e_{2}}\right]\left(v(t, x)-v^{*}\right)^{2}\right] d x .
\end{aligned}
$$

From (11) and considering that $u(s ; x)$ is a solution (2), one obtains, by adapting the writings, an expression of $\frac{\partial u(s, x)}{\partial s}$ similar to the first equation of (21). Therefore, $T_{12}$ becomes

$$
\begin{aligned}
T_{12}= & \int_{\Omega} \frac{v^{*}}{M_{v}^{\epsilon}}\left(u(t, x)-u^{*}\right) \\
& \times \int_{t-r_{1}}^{t}\left[\Delta u(s, x)+u(s, x)\left(-\left(u\left(s-r_{1}, x\right)-u^{*}\right),\right.\right. \\
& \left.\left.+\frac{a v(s, x)}{\left(u^{*}+e_{1}\right)\left(u(s, x)+e_{1}\right)}\left(u(s, x)-u^{*}\right)-\frac{a}{u^{*}+e_{1}}\left(v(s, x)-v^{*}\right)\right)\right] d s d x .
\end{aligned}
$$


So,

$$
\begin{aligned}
T_{12}= & \int_{\Omega} \frac{v^{*}}{M_{v}^{\epsilon}}\left(u(t, x)-u^{*}\right) \int_{t-r_{1}}^{t} \Delta u(s, x) d s d x \\
& +\int_{\Omega} \frac{v^{*}}{M_{v}^{\epsilon}}\left(u(t, x)-u^{*}\right) \int_{t-r_{1}}^{t} u(s, x)\left(-\left(u\left(s-r_{1}, x\right)-u^{*}\right)\right. \\
& \left.+\frac{a v(s, x)}{\left(u^{*}+e_{1}\right)\left(u(s, x)+e_{1}\right)}\left(u(s, x)-u^{*}\right)-\frac{a}{u^{*}+e_{1}}\left(v(s, x)-v^{*}\right)\right) d s d x
\end{aligned}
$$

Let us pose $T_{12}=\Upsilon+\Theta$, with $\Upsilon=\int_{\Omega} \frac{v^{*}}{M_{v}^{\epsilon}}\left(u(t, x)-u^{*}\right) \int_{t-r_{1}}^{t} \Delta u(s, x) d s d x$ and

$$
\begin{aligned}
\Theta= & \frac{v^{*}}{M_{v}^{\epsilon}} \int_{\Omega} \int_{t-r_{1}}^{t}\left(u(t, x)-u^{*}\right) u(s, x)\left(-\left(u\left(s-r_{1}, x\right)-u^{*}\right)\right. \\
& \left.\left.+\frac{a v(s, x)}{\left(u^{*}+e_{1}\right)\left(u(s, x)+e_{1}\right)}\left(u(s, x)-u^{*}\right)-\frac{a}{u^{*}+e_{1}}\left(v(s, x)-v^{*}\right)\right)\right] d s d x .
\end{aligned}
$$

From Green's formula $\Upsilon$ becomes

$$
\begin{aligned}
\Upsilon= & -\frac{v^{*}}{M_{v}^{\epsilon}} \int_{\Omega} \int_{t-r_{1}}^{t} \nabla u(s, x) \cdot \nabla\left(u(t, x)-u^{*}\right) d s d x \\
& +\frac{v^{*}}{M_{v}^{\epsilon}} \int_{t-r_{1}}^{t} \int_{\partial \Omega} \frac{\partial u(s, x)}{\partial n}\left(u(t, x)-u^{*}\right) d x d s .
\end{aligned}
$$

So, $\Upsilon=-\frac{v^{*}}{M_{v}^{\epsilon}} \int_{\Omega} \int_{t-r_{1}}^{t} \nabla u(s, x) \cdot \nabla\left(u(t, x)-u^{*}\right) d s d x$ because $\frac{\partial u(s, x)}{\partial n}=0$. Therefore, $\Upsilon=-\frac{v^{*}}{M_{v}^{\epsilon}} \int_{t-r_{1}}^{t} \int_{\Omega} \nabla u(s, x) \cdot \nabla u(t, x) d x d s$ because $\nabla u^{*}=0$. Consequently, $\Upsilon \leq \frac{v^{*}}{M_{v}^{\epsilon}} \times$ $\int_{t-r_{1}}^{t} \int_{\Omega} \frac{|\nabla u(t, x)|^{2}}{2}+\frac{|\nabla u(s, x)|^{2}}{2} d x d s$. So,

$$
\Upsilon \leq \frac{v^{*}}{M_{v}^{\epsilon}} r_{1} \int_{\Omega} \frac{|\nabla u(t, x)|^{2}}{2} d x+\frac{v^{*}}{M_{v}^{\epsilon}} \int_{t-r_{1}}^{t} \int_{\Omega} \frac{|\nabla u(s, x)|^{2}}{2} d x d s
$$

Let us compute the upper value of $\Theta$. One has

$$
\begin{aligned}
\Theta \leq & \frac{v^{*}}{M_{v}^{\epsilon}} \int_{\Omega} \int_{t-r_{1}}^{t} u(s, x)\left[\left(u(t, x)-u^{*}\right)^{2}+\left(u\left(s-r_{1}, x\right)-u^{*}\right)^{2}\right. \\
& +\frac{a v(s, x)}{2\left(u^{*}+e_{1}\right)\left(u(s, x)+e_{1}\right)}\left(\left(u(t, x)-u^{*}\right)^{2}+\left(u(s, x)-u^{*}\right)^{2}\right) \\
& \left.+\frac{a}{2\left(u^{*}+e_{1}\right)}\left(\left(u(t, x)-u^{*}\right)^{2}+\left(v(s, x)-v^{*}\right)^{2}\right)\right] d s d x
\end{aligned}
$$

So, by using the boundary of the solution $(u(s, x) ; v(s, x))$, one obtains

$$
\begin{aligned}
\Theta \leq & \int_{\Omega} \int_{t-r_{1}}^{t} \frac{v^{*}}{2 M_{v}^{\epsilon}} M_{u}^{\epsilon}\left[\left(u(t, x)-u^{*}\right)^{2}+\left(u\left(s-r_{1}, x\right)-u^{*}\right)^{2}\right] d s d x \\
& +\int_{\Omega} \int_{t-r_{1}}^{t} \frac{M_{u}^{\epsilon}}{2 e_{1}}\left(u(t, x)-u^{*}\right)^{2} d s d x+\int_{\Omega} \int_{t-r_{1}}^{t} \frac{M_{u}^{\epsilon}}{2 e_{1}}\left(u(s, x)-u^{*}\right)^{2} d s d x \\
& +\int_{\Omega} \int_{t-r_{1}}^{t} \frac{M_{u}^{\epsilon}}{2 M_{v}^{\epsilon}}\left(u(t, x)-u^{*}\right)^{2} d s d x+\int_{\Omega} \int_{t-r_{1}}^{t} \frac{M_{u}^{\epsilon}}{2 M_{v}^{\epsilon}}\left(v(s, x)-v^{*}\right)^{2} d s d x .
\end{aligned}
$$


Nindjin et al. Advances in Difference Equations （2018） 2018:177

Page 13 of 17

Finally,

$$
\begin{aligned}
\Theta \leq & \int_{\Omega} r_{1}\left(\frac{M_{u}^{\epsilon}}{2}+\frac{M_{u}^{\epsilon}}{2 e_{1}}+\frac{M_{u}^{\epsilon}}{2 M_{v}^{\epsilon}}\right)\left(u(t, x)-u^{*}\right)^{2} d s d x \\
& \left.+\int_{\Omega} \int_{t-r_{1}}^{t} \frac{M_{u}^{\epsilon}}{2}\left(u\left(s-r_{1}, x\right)-u^{*}\right)^{2}\right) d s d x \\
& +\int_{\Omega} \int_{t-r_{1}}^{t} \frac{M_{u}^{\epsilon}}{2 e_{1}}\left(u(s, x)-u^{*}\right)^{2} d s d x+\int_{\Omega} \int_{t-r_{1}}^{t} \frac{M_{u}^{\epsilon}}{2 M_{v}^{\epsilon}}\left(v(s, x)-v^{*}\right)^{2} d s d x .
\end{aligned}
$$

We use inequalities (22), (23), and (24) to complete the highest value of $\int_{\Omega} \frac{\partial l_{1}(u(t, x), v(t, x))}{\partial t} d x$. Because, as said earlier, $\int_{\Omega} \frac{\partial l_{1}(u(t, x), v(t, x))}{\partial t} d x=T_{11}+\Upsilon+\Theta+T_{2}$. Then posing $\Gamma=$ $\int_{\Omega} \frac{\partial l_{1}(u(t, x), v(t, x))}{\partial t} d x$, we have

$$
\begin{aligned}
\Gamma \leq & \left.-\frac{u^{*} v^{*}}{M_{v}^{\epsilon}} \int_{\Omega} \frac{|\nabla u(t, x)|^{2}}{u(t, x)^{2}} d x-\delta \frac{\left(v^{*}\right)^{2}}{b M_{v}^{\epsilon}} \int_{\Omega} \frac{|\nabla v(t, x)|^{2}}{v(t, x)^{2}}\right] d x \\
& +\int_{\Omega}\left[-\frac{e_{2}}{M_{v}^{\epsilon}}+\frac{1}{e_{1}}+\frac{1}{2}+\frac{1}{2 e_{2}}\right]\left(u(t, x)-u^{*}\right)^{2} d x \\
& +\int_{\Omega}\left[\left(-\frac{1}{M_{v}^{\epsilon}}+\frac{1}{2}+\frac{1}{2 e_{2}}\right)\left(v(t, x)-v^{*}\right)^{2}\right] d x \\
& +\frac{v^{*}}{M_{v}^{\epsilon}} r_{1} \int_{\Omega} \frac{|\nabla u(t, x)|^{2}}{2} d x+\frac{v^{*}}{M_{v}^{\epsilon}} \int_{t-r_{1}}^{t} \int_{\Omega} \frac{|\nabla u(s, x)|^{2}}{2} d x d s \\
& +\int_{\Omega} r_{1}\left(\frac{M_{u}^{\epsilon}}{2}+\frac{M_{u}^{\epsilon}}{2 e_{1}}+\frac{M_{u}^{\epsilon}}{2 M_{v}^{\epsilon}}\right)\left(u(t, x)-u^{*}\right)^{2} d x \\
& \left.+\int_{\Omega} \int_{t-r_{1}}^{t} \frac{M_{u}^{\epsilon}}{2}\left(u\left(s-r_{1}, x\right)-u^{*}\right)^{2}\right) d s d x \\
& +\int_{\Omega} \int_{t-r_{1}}^{t} \frac{M_{u}^{\epsilon}}{2 e_{1}}\left(u(s, x)-u^{*}\right)^{2} d s d x+\int_{\Omega} \int_{t-r_{1}}^{t} \frac{M_{u}^{\epsilon}}{2 M_{v}^{\epsilon}}\left(v(s, x)-v^{*}\right)^{2} d s d x .
\end{aligned}
$$

Hence

$$
\begin{aligned}
\Gamma \leq & \int_{\Omega}\left[|\nabla u(t, x)|^{2}\left(-\frac{v^{*} u^{*}}{M_{v}^{\epsilon} u(t, x)^{2}}+\frac{v^{*} r_{1}}{2 M_{v}^{\epsilon}}\right)-\delta|\nabla v(t, x)|^{2}\left(\frac{\left(v^{*}\right)^{2}}{b M_{v}^{\epsilon} v(t, x)^{2}}\right)\right] d x \\
& +\int_{\Omega}\left[-\frac{e_{2}}{M_{v}^{\epsilon}}+\frac{1}{e_{1}}+\frac{1}{2}+\frac{1}{2 e_{2}}+r_{1}\left(\frac{M_{u}^{\epsilon}}{2}+\frac{M_{u}^{\epsilon}}{2 e_{1}}+\frac{M_{u}^{\epsilon}}{2 M_{v}^{\epsilon}}\right)\right]\left(u(t, x)-u^{*}\right)^{2} d x \\
& +\int_{\Omega}\left[-\frac{1}{M_{v}^{\epsilon}}+\frac{1}{2}+\frac{1}{2 e_{2}}\right]\left(v(t, x)-v^{*}\right)^{2} d x \\
& \left.+\int_{\Omega} \int_{t-r_{1}}^{t} \frac{M_{u}^{\epsilon}}{2}\left(u\left(s-r_{1}, x\right)-u^{*}\right)^{2}\right) d s d x \\
& +\int_{\Omega} \int_{t-r_{1}}^{t} \frac{M_{u}^{\epsilon}}{2 e_{1}}\left(u(s, x)-u^{*}\right)^{2} d s d x+\int_{\Omega} \int_{t-r_{1}}^{t} \frac{M_{u}^{\epsilon}}{2 M_{v}^{\epsilon}}\left(v(s, x)-v^{*}\right)^{2} d s d x \\
& +\frac{v^{*}}{M_{v}^{\epsilon}} \int_{t-r_{1}}^{t} \int_{\Omega} \frac{|\nabla u(s, x)|^{2}}{2} d x d s .
\end{aligned}
$$


Now let us focus on $\frac{\partial \Sigma(t)}{\partial t}=\int_{\Omega} \frac{\partial \Sigma_{1}(t, x)}{\partial t} d x$. From Lemma 5.1 we have

$$
\begin{aligned}
\frac{\partial \Sigma(t)}{\partial t}= & \left.\int_{\Omega} \frac{r_{1} M_{u}^{\epsilon}}{2}\left(u\left(t-r_{1}, x\right)-u^{*}\right)^{2} d x-\int_{\Omega} \int_{t-r_{1}}^{t} \frac{M_{u}^{\epsilon}}{2}\left(u\left(s-r_{1}, x\right)-u^{*}\right)^{2}\right) d s d x \\
& +\int_{\Omega} \frac{r_{1} M_{u}^{\epsilon}}{2 e_{1}}\left(u(t, x)-u^{*}\right)^{2} d x-\int_{\Omega} \int_{t-r_{1}}^{t} \frac{M_{u}^{\epsilon}}{2 e_{1}}\left(u(s, x)-u^{*}\right)^{2} d s d x \\
& +\int_{\Omega} \frac{r_{1} M_{u}^{\epsilon}}{2 M_{v}^{\epsilon}}\left(v(t, x)-v^{*}\right)^{2} d x-\int_{\Omega} \int_{t-r_{1}}^{t} \frac{M_{u}^{\epsilon}}{2 M_{v}^{\epsilon}}\left(v(s, x)-v^{*}\right)^{2} d s d x \\
& +\int_{\Omega} \frac{r_{1} M_{u}^{\epsilon}}{2}\left(u(t, x)-u^{*}\right)^{2} d x-\int_{\Omega} \frac{r_{1} M_{u}^{\epsilon}}{2}\left(u\left(t-r_{1}, x\right)-u^{*}\right)^{2} d x \\
& +\frac{r_{1} v^{*}}{M_{v}^{\epsilon}} \int_{\Omega} \frac{|\nabla u(t, x)|^{2}}{2} d x-\frac{v^{*}}{M_{v}^{\epsilon}} \int_{\Omega} \int_{t-r_{1}}^{t} \frac{|\nabla u(s, x)|^{2}}{2} d s d x .
\end{aligned}
$$

Consequently, taking into account the highest value of $\frac{\partial \Sigma(t, x)}{\partial t}$ and $\int_{\Omega} \frac{\partial l_{1}(u(t, x), v(t, x))}{\partial t} d x$, we obtain

$$
\begin{aligned}
\frac{d L}{d t} \leq & \int_{\Omega}|\nabla u(t, x)|^{2}\left(-\frac{v^{*} u^{*}}{M_{v}^{\epsilon} u(t, x)^{2}}+\frac{v^{*} r_{1}}{2 M_{v}^{\epsilon}}+\frac{r_{1} v^{*}}{2 M_{v}^{\epsilon}}\right) d x \\
& -\int_{\Omega} \delta|\nabla v(t, x)|^{2}\left(\frac{\left(v^{*}\right)^{2}}{b M_{v}^{\epsilon} v(t, x)^{2}}\right) d x \\
& +\int_{\Omega}\left[-\frac{e_{2}}{M_{v}^{\epsilon}}+\frac{1}{e_{1}}+\frac{1}{2}+\frac{1}{2 e_{2}}+r_{1}\left(\frac{M_{u}^{\epsilon}}{2}+\frac{M_{u}^{\epsilon}}{2 e_{1}}+\frac{M_{u}^{\epsilon}}{2 M_{v}^{\epsilon}}\right)+\frac{r_{1} M_{u}^{\epsilon}}{2}+\frac{r_{1} M_{u}^{\epsilon}}{2 e_{1}}\right] \\
& \times\left(u(t, x)-u^{*}\right)^{2} d x \\
& \left.+\int_{\Omega}\left[-\frac{1}{M_{v}^{\epsilon}}+\frac{1}{2}+\frac{1}{2 e_{2}}+\frac{r_{1} M_{u}^{\epsilon}}{2 M_{v}^{\epsilon}}\right]\left(v(t, x)-v^{*}\right)^{2}\right] d x .
\end{aligned}
$$

So,

$$
\begin{aligned}
\frac{d L}{d t} \leq & \int_{\Omega}|\nabla u(t, x)|^{2}\left(-\frac{v^{*} u^{*}}{M_{v}^{\epsilon} u(t, x)^{2}}+\frac{v^{*} r_{1}}{M_{v}^{\epsilon}}\right) d x-\int_{\Omega} \delta|\nabla v(t, x)|^{2}\left(\frac{\left(v^{*}\right)^{2}}{b M_{v}^{\epsilon} v(t, x)^{2}}\right) d x \\
& +\int_{\Omega}\left[-\frac{e_{2}}{M_{v}^{\epsilon}}+\frac{1}{e_{1}}+\frac{1}{2}+\frac{1}{2 e_{2}}+r_{1}\left(M_{u}^{\epsilon}+\frac{M_{u}^{\epsilon}}{e_{1}}+\frac{M_{u}^{\epsilon}}{2 M_{v}^{\epsilon}}\right)\right]\left(u(t, x)-u^{*}\right)^{2} d x \\
& \left.+\int_{\Omega}\left[-\frac{1}{M_{v}^{\epsilon}}+\frac{1}{2}+\frac{1}{2 e_{2}}+\frac{r_{1} M_{u}^{\epsilon}}{2 M_{v}^{\epsilon}}\right]\left(v(t, x)-v^{*}\right)^{2}\right] d x .
\end{aligned}
$$

That is why

$$
\begin{aligned}
\frac{d L}{d t} \leq & K_{1} \int_{\Omega}|\nabla u(t, x)|^{2} d x \\
& -\frac{\delta\left(m_{v}^{\epsilon}\right)^{2}}{b\left(M_{v}^{\epsilon}\right)^{3}} \int_{\Omega}|\nabla v(t, x)|^{2} d x+K_{2} \int_{\Omega}\left(u(t, x)-u^{*}\right)^{2} d x \\
& +K_{3} \int_{\Omega}\left(v(t, x)-v^{*}\right)^{2} d x
\end{aligned}
$$


with

$$
\begin{aligned}
& K_{1}=\frac{v^{*}}{M_{v}^{\epsilon}}\left(r_{1}-\frac{u^{*}}{\left(M_{u}^{\epsilon}\right)^{2}}\right), \\
& K_{2}=-\frac{e_{2}}{M_{v}^{\epsilon}}+\frac{1}{e_{1}}+\frac{1}{2}+\frac{1}{2 e_{2}}+r_{1}\left(M_{u}^{\epsilon}+\frac{M_{u}^{\epsilon}}{e_{1}}+\frac{M_{u}^{\epsilon}}{2 M_{v}^{\epsilon}}\right), \\
& K_{3}=-\frac{1}{M_{v}^{\epsilon}}+\frac{1}{2}+\frac{1}{2 e_{2}}+\frac{r_{1} M_{u}^{\epsilon}}{2 M_{v}^{\epsilon}} .
\end{aligned}
$$

Under the conditions and assumptions of Theorem 5.1 and for a sufficiently small $r_{1}$ delay, $K_{1}, K_{2}$, and $K_{3}$ are all inferior to zero. Indeed, by making $\epsilon$ tend towards $0, M_{\nu}^{\epsilon}$ tend towards $M_{v}=e^{r_{1}}+e_{2}$. Since $r_{1}$ is smallest, there is $r_{0}$ from which $K_{1}<0$. However, by hypothesis one has $e_{1}<\frac{2 a e_{2}}{e_{2}+1}$, which implies

$$
\frac{1}{2}+\frac{1}{2 e_{2}}<\frac{a}{e_{1}}<\frac{1}{M_{v}}
$$

So, $K_{3}$ is negative. By multiplying inequalities (30) by $e_{2}$, one has $\frac{e_{2}}{2}+\frac{1}{2}<\frac{e_{2}}{M_{v}^{\epsilon}}$. Yet, by hypothesis, one has $\frac{2 e_{2}}{e_{2}^{2}-1}<e_{1}$.

So, $\frac{1}{e_{1}}+\frac{1}{2}+\frac{1}{2 e_{2}}<\frac{e_{2}}{2}+\frac{1}{2}<\frac{e_{2}}{M_{v}^{\epsilon}}$. Consequently, $K_{2}$ is negative. In this case,

$$
\frac{d L}{d t}<0
$$

Hence the interior equilibrium point $\left(u^{*}, v^{*}\right)$ of system (1) is globally asymptotically stable.

Remark 5.1 Let $r_{0}$ be the only solution of the equation $r_{1}-\frac{u^{*}}{e^{2 r_{1}}}=0$.

If $e_{1}-a e_{2}>a$ and (15) is verified then, for all $r_{1}<\min \left\{r_{0} ; \ln \left(\frac{e_{1}-a e_{2}}{a}\right)\right\}$, the interior equilibrium point $\left(u^{*}, v^{*}\right)$ is globally asymptotically stable.

\section{Numerical simulations}

Let us consider the space $\bar{\Omega}=[0 ; 8.4] \times[0 ; 8.4]$, the period $T=60$, and the following control parameters: $a=0.69, b=0.2, \delta=1, e_{1}=3.5, e_{2}=4, \mu_{i}=\frac{2 \pi^{2}}{(8.4)^{2}}$.

For the graphic illustration, let us consider the initial conditions $\left(u_{0}, v_{0}\right)=(0.5 ; 0.3)$, the step $d t=h=0.1, d x=d y=0.4$, and the number iterations of time $N=600$. From condition (3) of Theorem 2.1, the system is permanent if $0<r_{1}<r_{\lim }=0.3254$. For $r_{1}=0.3$, one obtains the maximum and minimum values of $u$ and $v: u_{\min }=0.0043, u_{\max }=1.3499, v_{\min }=$ $4, v_{\max }=5.3499$. The trivial equilibrium points of the model are $S_{0}=(0 ; 0), S_{1}=(0 ; 4), S_{2}=$ $(1 ; 0)$. In addition, $S_{3}=(0.2636 ; 4.2636)$ is the unique interior equilibrium point verifying condition 15 of Theorem 5.1. Then $S_{3}$ is globally stable. Hence, illustrating figure (Fig. 1) for the space position $x=1.2$ and $y=0.8$.

- Interpretation: The trajectories of prey $u$ and predators $v$ stabilize respectively around 0.2636 and 4.2636 when $t$ is greater than 40 . As for the orbits, they are moving away from trivial equilibrium points and converge towards $S_{3}=(0.2636 ; 4.2636)$. Hence, $S_{3}$ is globally stable, which is illustrated by the second and the third figure. It is noticed that for values $x$ and $y$ in $\Omega$, and for all initial conditions $u_{0}$ and $v_{0}$ strictly positive, the orbits and trajectories are similar to those of Fig. 1. That is in line with the theoretical results. 


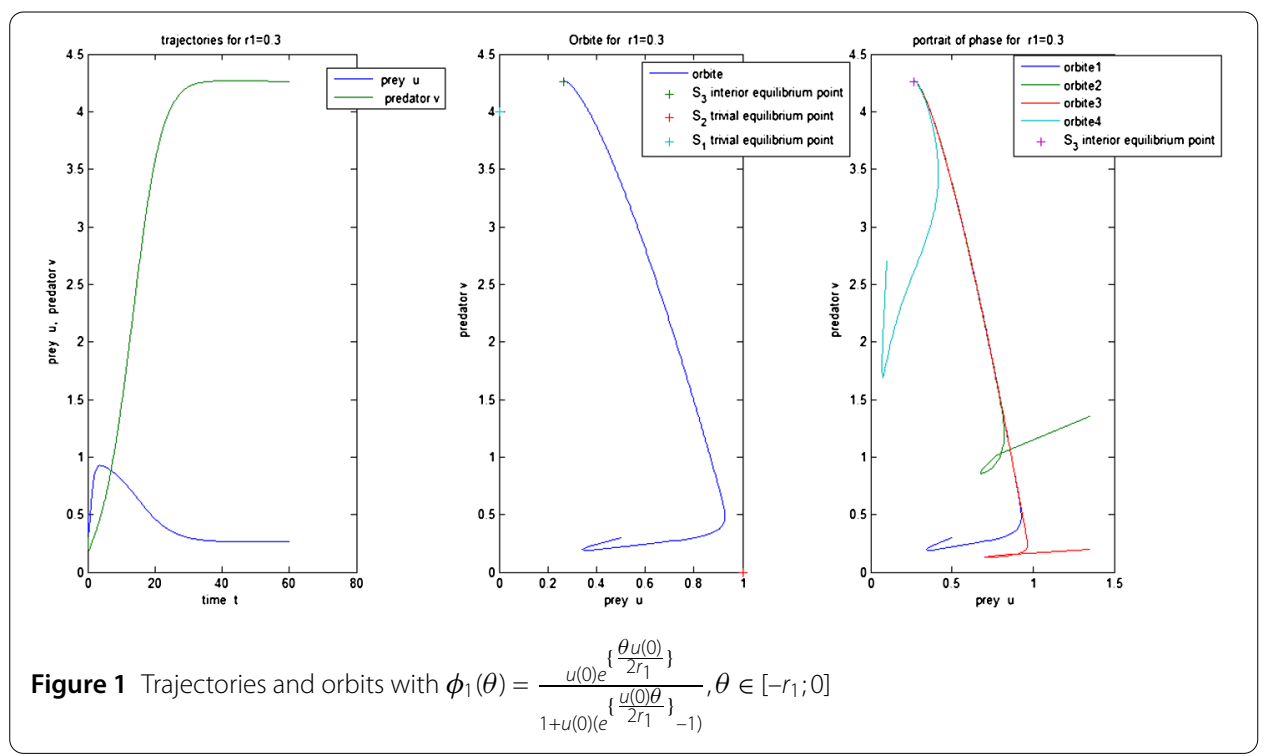

\section{Conclusion}

In this article, we have shown that the solutions of the model considered are globally bounded. The local stability study revealed that the delay introduced in the feedback of the equation prey, which is essentially due to internal competition between preys, has a real impact on the stability of the indoor fixed point $\left(u^{*}, v^{*}\right)$. In effect, from a certain threshold of delay $r_{1}$, noted $r_{1}^{*}$, a change of stability of this unique positive internal fixed point is observed. We have built an appropriate Lyapunov's functional to study the global stability of the fixed point $\left(u^{*}, v^{*}\right)$. We retain that for a smallest length of the delay $r_{1}$ and for an internal competition raised between the preys, this fixed point is globally asymptotically stable. This conclusion seems to be in line with reality. The faster preys reach maturity, the better they participate in the internal competition between their species. This competition makes the interactions of all the species involved more dynamic.

For future works, we will firstly extend the method used for studying the global stability to a food chain better accomplished with several species in interaction. Secondly we will not only introduce many delays in this model, but also study its impact on the analysis of dynamic stability. Finally we will study other problems of the dynamic which includes diffusive terms with the same methods.

\section{Acknowledgements}

The authors wish to thank in advance the editor and the referees who will read this manuscript.

Competing interests

The authors declare that they have no competing interests.

Authors' contributions

The author AFN is the head of the group "Dynamic of Population" to which Messrs. KTT, HO, and AT belong. Messrs KTT and AT are his students, while Mr HO is his assistant. As such, it is AFN who guides, animates the research and validates the results obtained. In this framework, he helped to improve some results like those of local stability and the sufficient conditions that give the global stability. The author KTT proposed and justified the basic model of this paper. The authors $\mathrm{HO}$ and KTT conducted all the calculations of this paper; at the level of the global boundedness, local and global stability and permanence. Author AT inspired the coupled use of Lyapunov functional Sects. 1 and 2. This method allowed KTT, AT, and $\mathrm{HO}$ to make the calculations and estimates in order to identify sufficient conditions leading to overall stability. All authors read and approved the final manuscript. 


\section{Publisher's Note}

Springer Nature remains neutral with regard to jurisdictional claims in published maps and institutional affiliations.

Received: 20 December 2017 Accepted: 26 April 2018 Published online: 10 May 2018

\section{References}

1. Camara, B.I, Aziz-Alaoui, M.A.: Complexity in a prey predator model. ARIMA 9, 109-122 (2008)

2. Nindjin, A.F., Aziz-Alaoui, M.A., Cadivel, M.: Analysis of predator-prey model with modified Leslie-Gower and Holling-type II schemes with time delay. Nonlinear Anal., Real World Appl. 7, 1104-1118 (2006)

3. Tian, Y., Weng, P.: Asymptotic stability of diffusive predator-prey model with modified Leslie-Gower and Holling-type II schemes and nonlocal time delays. IMA J. Math. Control Inf. 31, 1-14 (2013). https://doi.org/10.1093/imamci/dns041

4. Tian, Y.: Stability for a diffusive delayed predator-prey model with modified Leslie-Gower and Holling-type II schemes. Appl. Math. 2, 217-240 (2014)

5. Pao, C.V.: Dynamics of nonlinear parabolic systems with time delay. J. Math. Anal. Appl. 198, 751-779 (1996)

6. Meng, X., Wang, L., Zhang, T.: Global dynamics analysis of a nonlinear impulsive stochastic chemostat system in a polluted environment. J. Appl. Anal. Comput. 6(3), 865-875 (2016)

7. Meng, X., Zhao, S., Feng, T.: Dynamics of a novel nonlinear stochastic SIS epidemic model with double epidemic hypothesis. J. Math. Anal. Appl. 433(1), 227-242 (2016)

8. Zhang, T., Ma, W., Meng, X., Zhang, T.: Periodic solution of a prey-predator model with nonlinear state feedback control. Appl. Math. Comput. 266, 95-107 (2015)

9. Menga, X., Zhao, S., Zhang, W.: Adaptive dynamics analysis of a predator-prey model with selective disturbance. Appl. Math. Comput. 266, 946-958 (2015)

\section{Submit your manuscript to a SpringerOpen ${ }^{\mathcal{O}}$ journal and benefit from:}

- Convenient online submission

- Rigorous peer review

- Open access: articles freely available online

- High visibility within the field

- Retaining the copyright to your article

Submit your next manuscript at $\boldsymbol{~ s p r i n g e r o p e n . c o m ~}$ 